\title{
Totally Contact Umbilical Radical Transversal Lightlike Submanifold of an Almost Contact Manifold With $B$-Metric
}

\author{
Anu Devgan ${ }^{1}$ and R. K. Nagaich ${ }^{2}$ \\ ${ }^{1}$ Department of Mathematics, Punjabi University, Patiala-147002, India. \\ anudevgan13pup@gmail.com \\ ${ }^{2}$ Department of Mathematics, Punjabi University, Patiala-147002, India. \\ nagaich58rakesh@gmail.com
}

\section{ABSTRACT:}

In the present paper, we study the geometry of totally contact umbilical radical transversal lightlike submanifolds and totally contact umbilical $C R$-submanifold of an indefinite Sasaki-like almost contact manifold with $B$-metric. We investigate the necessary and sufficient condition for the characterization of the induced connection to be a metric connection. Finally, we have proved that for a totally contact umbilical $C R$-submanifold, totally contact umbilical radical transversal lightlike submanifold is a totally geodesic radical transversal lightlike submanifold.

Key words : Almost contact manifolds; B-metric; Totally umbilical; Radical transversal lightlike submanifolds.

\section{AMS Mathematics Subject Classification(2010): 53C15, 53C40, 53 C50.}

\section{INTRODUCTION}

Yano and Kon[12] introduced the geometry of contact $C R$ submanifolds of a Sasakian manifold (odd dimensional manifold). Further, Matsumoto[8] studied totally umbilical submanifolds of contact $C R$ submanifolds. Since contact geometry has its wider applications in the theory of differential equations, optics and phase spaces of a dynamical system, therefore it becomes an impotant topic of discussion. Ganchev et al. [7] initiated the study of odd dimensional manifolds known as an almost contact manifolds with $B$-metric which serves as a natural analogue of the geometry of the almost complex manifolds with Norden metric.

The lightlike submanifolds were introduced and studied by Duggal and Bejancu[4]. Recently, Yildirim and Sahin[11] introduce the notion of radical transversal lightlike submanifolds of an indefinite Sasakian manifold and obtained many interesting results. Nakova[10], clubbed together the geometry of $C R$-submanifolds and lightlike submanifolds of almost complex manifold with Norden metric. This motivated us to club these geometries in odd dimensional case.

The growing importance of lightlike submanifolds in mathematical physics, especially in relativity, motivated us to study radical transversal lightlike submanifolds extensively. Here, we introduce and study totally contact umbilical radical transversal lightlike submanifolds of Sasaki-like almost contact manifolds with $B$-metric.

The paper is arranged as follows. In section 2, we recall some definitions for almost contact manifolds with $B$-metric and give basic information on the lightlike geometry required for this paper. Section 3 is devoted to the study of the geometry of totally contact umbilical radical transversal lightlike submanifolds and totally contact $C R$-submanifolds. In section 4 , we give geometric conditions for the induced connection to become a metric connection and further proved that for a totally contact umbilical $C R$-submanifold, the totally contact umbilical radical transversal lightlike submanifold becomes totally geodesic submanifold.

\section{PRELIMINARIES}

\subsection{Almost Contact Manifolds with $B$-metric}

Let $\left(\bar{M}^{2 n+1}, \varphi, \zeta, \eta\right)$ be an almost contact manifold with $B$-metric $\bar{g}$, i.e. an almost contact structure $(\varphi, \zeta, \eta)$ consisting of a tensor field $\varphi$ of type $(1,1)$ a vector field $\zeta$ and a 1 -form $\eta$ on $\bar{M}$ satisfying the following algebraic conditions :

$$
\varphi^{2}(X)=-X+\eta(X) \zeta, \quad \eta(\zeta)=1, \quad \varphi \zeta=0, \quad \eta \circ \zeta=0
$$

An almost contact manifold $(\bar{M}, \varphi, \zeta, \eta)$ admits a semi-Riemannian metric $\bar{g}$ such that[7]

$$
\bar{g}(\varphi X, \varphi Y)=-\bar{g}(X, Y)+\varepsilon \eta(X) \eta(Y), \quad \varepsilon= \pm 1,
$$

for arbitrary vector fields $X$ and $Y$ on $\bar{M}$. 
The associated metric $\overline{\widetilde{g}}$ of $\bar{g}$ on $\bar{M}$ is defined by

$$
\overline{\widetilde{g}}(X, Y)=\bar{g}(\bar{\varphi} X, Y)+\eta(X) \eta(Y),
$$

for arbitrary vector fields $X$ and $Y$ on $\bar{M}$ and $\overline{\widetilde{g}}$ is also a $B$ - metric on $\bar{M}$. The manifold $(\bar{M}, \varphi, \zeta, \eta, \overline{\widetilde{g}})$ is also called an almost contact manifold with $B$-metric. Both the metrics $\bar{g}$ and $\overline{\widetilde{g}}$ are indefinite of signature $(n+1, n)$.

The following corollaries are valid for an almost contact manifold with $B$-metric :

$$
\eta(X)=g(X, \zeta), \quad g(\varphi X, Y)=g(X, \varphi Y) .
$$

Let $\bar{\nabla}$ and $\overline{\widetilde{\nabla}}$ denote the Levi-Civita connection for the metric $\bar{g}$ and $\overline{\widetilde{g}}$ respectively. The tensor field $F$ of type $(0,3)$ on $\bar{M}$ is defined as

$$
F(X, Y, Z)=\bar{g}\left(\left(\bar{\nabla}_{X} \varphi\right) Y, Z\right)
$$

and the following general properties holds[7] :

$$
F(X, Y, Z)=F(X, Z, Y)=F(X, \varphi Y, \varphi Z)=F(X, Y, Z)+\eta(Y) F(X, \zeta, Z)+\eta(Z) F(X, Y, \zeta),
$$

for any $X, Y, Z \in \Gamma(T \bar{M})$. The relations of $F$ with $\nabla \zeta$ and $\nabla \eta$ are given by :

$$
\left(\nabla_{X} \eta\right) Y=g\left(\nabla_{X} \zeta, Y\right)=F(X, \varphi Y, \zeta), \quad \eta\left(\nabla_{X} \zeta\right)=0, \quad \varphi\left(\nabla_{X} \varphi\right) \zeta=\nabla_{X} \zeta
$$

Let $\left\{e_{i}, \zeta\right\},(i=1,2, \ldots . .2 n)$ be a basis of $T_{m} M$ and $\left(g^{i j}\right)$ be the inverse matrix of $g_{i j}$ then for $X \in \Gamma\left(T_{m} \bar{M}\right)$, the following 1 -forms are associated with $F$ :

$$
\theta(X)=g^{i j} F\left(e_{i}, e_{\varphi}, X\right), \quad \theta^{*}(X)=g^{i j} F\left(e_{i}, \varphi e_{\varphi}, X\right), \quad \omega(X)=F(\zeta, \zeta, X),
$$

Using above equation, we have

$$
\omega(\zeta)=0 \quad \theta^{*}(\varphi X)=-\theta\left(\varphi^{2} X\right)-\omega(X) .
$$

An almost contact structure $(\varphi, \zeta, \eta)$ is said to be normal if and only if Nijenhuis tensor denoted by $N$ vanishes[3] and the manifold $(M, \varphi, \zeta, \eta, g)$ is called normal almost contact manifold. The Nijenhuis tensor $N$ of the almost contact structure is defined by

$$
\begin{gathered}
N=[\varphi, \varphi]+d \eta \otimes \zeta, \\
N(X, Y)=\varphi^{2}[X, Y]+[\varphi X, \varphi Y]-\varphi[\varphi X, Y]-\varphi[X, \varphi Y]+d \eta \otimes \zeta .
\end{gathered}
$$

In [7] Ganchev et al. defined eleven basic classes $F_{i}(i=1,2, \ldots .11)$ of almost contact manifolds with $B$-metric and gave a classification of almost contact manifolds with $B$-metric with respect to tensor $F$. The special class $F_{0}$ defined by the condition $F(X, Y, Z)=0$ belongs to each of the basic classes. So, throughout this paper, we will consider the class $F_{0}$.

Definition 2.1.[9] An almost contact manifold $(\bar{M}, \varphi, \zeta, \eta, g)$ with $B$ - metric is known as Sasaki-like if the structure tensors $\varphi, \zeta, \eta, g$ satisfy the following equalities

$$
\begin{gathered}
F(X, Y, Z)=F(\zeta, Y, Z)=F(\zeta, \zeta, Z)=0, \\
F(X, Y, \zeta)=-g(X, Y) .
\end{gathered}
$$

Also, the covariant derivative $\bar{\nabla} \varphi$ satisfy the following equality 


$$
\left(\bar{\nabla}_{X} \varphi\right) Y=-\bar{g}(X, Y) \zeta-\eta(Y) X+2 \eta(X) \eta(Y) \zeta
$$

In this paper, we call these manifolds as indefinite Sasaki-like almost contact manifold with $B$-metric.

\subsection{Lightlike Submanifolds Of Semi-Riemannian Manifolds}

In this section we recall some basic notations and fundamental equations for lightlike submanifolds of semi-Riemannain manifolds. An $m$-dimensional submanifold $(M, \tilde{g})$ immersed in a real $(m+n)$-dimensional semi-Riemannian manifold $(\bar{M}, \overline{\widetilde{g}})$ of constant index $q$ such that $m, n \geq 1,1 \leq q \leq m+n-1$ and $\tilde{g}$ be the induced metric of $\overline{\widetilde{g}}$ on $M$. Then $M$ is called a lightlike submanifold of $\bar{M}$ if $\overline{\widetilde{g}}$ is degenerate metric on the tangent bundle $T M$ of $M$. For a degenerate metric $\tilde{g}$ on $M, T M^{\perp}$ is a degenerate $n$-dimensional subspace of $T_{x} \bar{M}$. Thus both $T_{x} M$ and $T_{x} M^{\perp}$ are no longer complementary but degenerate orthogonal subspaces of $T \bar{M}$. So, there exists a subspace called radical or null subspace, that is,

$$
\operatorname{Rad}\left(T_{x} M\right)=T_{x} M \cap T_{x} M^{\perp} .
$$

Further, if the mapping $\operatorname{Rad}(T M): x \in M \rightarrow \operatorname{RadT}_{x} M$, defines a smooth distribution of rank $r>0$ on $M$ then the submanifold $M$ is called an $r$-lightlike submanifold of $\bar{M}$ (for detail see [4]) and $\operatorname{Rad}(T M)$ is known as the radical distribution on $M$ and a semi-Riemannian complementary distribution $S(T M)$ of $\operatorname{Rad}(T M)$ in $T M$ is called Screen distribution, that is,

$$
T M=\operatorname{Rad} T M \perp S(T M),
$$

and $S\left(T M^{\perp}\right)$ is a complementary vector subbundle to $\operatorname{RadTM}$ in $T M^{\perp}$. Let $\operatorname{tr}(T M)$ and $\operatorname{ltr}(T M)$ be complementary (but not orthogonal) vector bundles to $T M$ in $\left.T \bar{M}\right|_{M}$ and to $\operatorname{RadTM}$ in $S\left(T M^{\perp}\right)^{\perp}$ respectively. Then, we have

$$
\begin{gathered}
\operatorname{tr}(T M)=\operatorname{ltr}(T M) \perp S\left(T M^{\perp}\right) . \\
\left.T \bar{M}\right|_{M}=T M \oplus \operatorname{tr}(T M)=(\operatorname{Rad} T M \oplus \operatorname{ltr}(T M)) \perp S(T M) \perp S\left(T M^{\perp}\right) . \\
T M=\operatorname{Rad}(T M) \perp S(T M)
\end{gathered}
$$

and $S\left(T M^{\perp}\right)$ is a complementary vector subbundle to $\operatorname{Rad}(T M)$ in $T M^{\perp}$. Let $\operatorname{tr}(T M)$ and $\operatorname{ltr}(T M)$ be complementary (but not orthogonal) vector bundles to $T M$ in $\left.T \bar{M}\right|_{M}$ and to $\operatorname{Rad}(T M)$ in $S\left(T M^{\perp}\right)^{\perp}$ respectively. Then, we have

$$
\begin{gathered}
\operatorname{tr}(T M)=\operatorname{ltr}(T M) \perp S\left(T M^{\perp}\right) . \\
\left.T \bar{M}\right|_{M}=T M \oplus \operatorname{tr}(T M)=(\operatorname{Rad}(T M) \oplus \operatorname{ltr}(T M)) \perp S(T M) \perp S\left(T M^{\perp}\right) .
\end{gathered}
$$

We have studied the following possible four cases with respect to the dimension $m$ and codimension $n$ of $\mathrm{M}$ and rank $r$ of RadTM :

1. $r$-lightlike, if $0<r<\min (m, n)$;

2. coisotropic, if $1 \leq r=n<m, S\left(T M^{\perp}\right)=\{0\}$;

3. isotropic, if $1<r=m<n, S(T M)=\{0\}$;

4. totally lightlike, if $1<r=m=n, S(T M)=\{0\}=S\left(T M^{\perp}\right)$.

Let $\mathrm{u}$ be a local coordinate neighborhood of $M$ and consider the local quasi-orthonormal fields of frames of $\bar{M}$ along $M$, on $\mathrm{u}$ as $\left\{\xi_{1}, \ldots, \xi_{r}, W_{r+1}, \ldots, W_{n}, N_{1}, \ldots, N_{r}, X_{r+1}, \ldots, X_{m}\right\}$, where $\left\{\xi_{1}, \ldots, \xi_{r}\right\},\left\{N_{1}, \ldots, N_{r}\right\}$ are local lightlike bases of $\Gamma\left(\left.\operatorname{RadTM}\right|_{\mathrm{u}}\right), \Gamma\left(\left.\operatorname{ltr}(T M)\right|_{\mathrm{u}}\right)$ and $\left\{W_{r+1}, \ldots, W_{n}\right\},\left\{X_{r+1}, \ldots, X_{m}\right\}$ are local orthonormal bases of 
$\Gamma\left(\left.S\left(T M^{\perp}\right)\right|_{\mathrm{u}}\right)$ and $\Gamma\left(\left.S(T M)\right|_{\mathrm{u}}\right)$, respectively. For this quasi-orthonormal fields of frames, we have the following theorem :

Theorem 2.1.([4]) Let $\left(M, g, S(T M), S\left(T M^{\perp}\right)\right)$ be an $r$-lightlike submanifold of a semi-Riemannian manifold $(\bar{M}, \bar{g})$. Then there exists a complementary vector bundle $\operatorname{ltr}(T M)$ of $\operatorname{Rad}(T M)$ in $S\left(T M^{\perp}\right)^{\perp}$ and a basis of $\Gamma\left(\left.\operatorname{ltr}(T M)\right|_{\mathrm{U}}\right)$ consisting of smooth section $\left\{N_{a}\right\}$ of $\left.S\left(T M^{\perp}\right)^{\perp}\right|_{\mathrm{U}}$, where $\mathrm{U}$ is a coordinate neighborhood of $M$, such that

$$
\bar{g}\left(N_{a}, \xi_{b}\right)=\delta_{a b}, \quad \bar{g}\left(N_{a}, N_{b}\right)=0, \quad \text { forany } a, b \in\{1,2, . ., r\},
$$

where $\left\{\xi_{1}, \ldots, \xi_{r}\right\}$ is a lightlike basis of $\Gamma(\operatorname{Rad}(T M))$.

Let $\bar{\nabla}$ denote the Levi-Civita connection on $\bar{M}$, then using the decomposition (2.2.3), the Gauss and Weingarten formulae are given as :

$$
\overline{\widetilde{\nabla}}_{X} Y=\tilde{\nabla}_{X} Y+h(X, Y), \quad \overline{\widetilde{\nabla}}_{X} U=-A_{U} X+\nabla_{X}^{\perp} U,
$$

for any $X, Y \in \Gamma(T M)$ and $U \in \Gamma(\operatorname{tr}(T M))$, where $\left\{\tilde{\nabla}_{X} Y, A_{U} X\right\}$ and $\left\{h(X, Y), \nabla_{X}^{\perp} U\right\}$ belong to $\Gamma(T M)$ and $\Gamma(\operatorname{tr}(T M))$, respectively. Here $\tilde{\nabla}$ is a torsion-free linear connection on $M, h$ is a symmetric bilinear form on $\Gamma(T M)$ which is called second fundamental form, $A_{U}$ is a linear a operator on $M$ and known as shape operator.

According to (2.2.2), considering the projection morphisms $L$ and $S$ of $\operatorname{tr}(T M)$ on $\operatorname{ltr}(T M)$ and $S\left(T M^{\perp}\right)$, respectively, then (2.2.4) becomes

$$
\overline{\widetilde{\nabla}}_{X} Y=\tilde{\nabla}_{X} Y+h^{l}(X, Y)+h^{s}(X, Y), \quad \overline{\widetilde{\nabla}}_{X} U=-A_{U} X+D_{X}^{l} U+D_{X}^{s} U,
$$

where we put

$$
\begin{array}{cc}
h^{l}(X, Y)=L(h(X, Y)), & h^{s}(X, Y)=S(h(X, Y)), \\
D_{X}^{l} U=L\left(\nabla_{X}^{\perp} U\right), & D_{X}^{s} U=S\left(\nabla_{X}^{\perp} U\right) .
\end{array}
$$

As $h^{l}$ and $h^{s}$ are $\Gamma(\operatorname{ltr}(T M))$-valued and $\Gamma\left(S\left(T M^{\perp}\right)\right)$-valued respectively, therefore these are known as the lightlike second fundamental form and the screen second fundamental form on $M$. In particular,

$$
\overline{\widetilde{\nabla}}_{X} N=-A_{N} X+\nabla_{X}^{l} N+D^{s}(X, N), \quad \bar{\nabla}_{X} W=-A_{W} X+\nabla_{X}^{s} W+D^{l}(X, W),
$$

where $X \in \Gamma(T M), N \in \Gamma(\operatorname{ltr}(T M))$ and $W \in \Gamma\left(S\left(T M^{\perp}\right)\right)$. Using (2.2.5)-( 2.2.6), we obtain

$$
\begin{gathered}
\overline{\widetilde{g}}\left(h^{s}(X, Y), W\right)+\overline{\widetilde{g}}\left(Y, D^{l}(X, W)\right)=\tilde{g}\left(A_{W} X, Y\right), \\
\overline{\widetilde{g}}\left(h^{l}(X, Y), \xi\right)+\overline{\widetilde{g}}\left(Y, h^{l}(X, \xi)\right)+\tilde{g}\left(Y, \nabla_{X} \xi\right)=0, \\
\overline{\widetilde{g}}\left(A_{W} X, N\right)=\overline{\widetilde{g}}\left(D^{s}(X, N), W\right), \\
\overline{\widetilde{g}}\left(A_{N} X, P Y\right)=\overline{\widetilde{g}}\left(N, \bar{\nabla}_{X} P Y\right),
\end{gathered}
$$

where $P$ is the projection morphism of $T M$ on $S(T M)$, for any $X, Y \in \Gamma(T M), \xi \in \Gamma(\operatorname{RadTM})$ and $W \in \Gamma\left(S\left(T M^{\perp}\right)\right)$. Using (2.2.1), we can induce some new geometric objects on the screen distribution $S(T M)$ on $M$ as

$$
\tilde{\nabla}_{X} P Y=\tilde{\nabla}_{X}^{*} P Y+h^{*}(X, P Y), \quad \tilde{\nabla}_{X} \xi=-A_{\xi}^{*} X+\nabla_{X}^{* t} \xi
$$

for any $X, Y \in \Gamma(T M)$ and $\xi \in \Gamma(\operatorname{RadTM})$, where $\left\{\tilde{\nabla}_{X}^{*} P Y, A_{\xi}^{*} X\right\}$ and $\left\{h^{*}(X, Y), \nabla_{X}^{* t} \xi\right\}$ belong to 
$\Gamma(S(T M))$ and $\Gamma(\operatorname{RadTM})$, respectively. $\nabla^{*}$ and $\nabla^{* t}$ are linear connections on complementary distributions $S(T M)$ and RadTM , respectively. $h^{*}$ and $A^{*}$ are $\Gamma(\operatorname{RadTM})$-valued and $\Gamma(S(T M))$-valued bilinear forms and $h^{*}$ is called the second fundamental form of screen distribution $S(T M)$. Using (2.2.5) and (2.2.11), we obtain

$$
\overline{\widetilde{g}}\left(h^{l}(X, P Y), \xi\right)=\tilde{g}\left(A_{\xi}^{*} X, P Y\right) \quad \text { and } \quad \overline{\widetilde{g}}\left(h^{*}(X, P Y), N\right)=\tilde{g}\left(A_{N} X, P Y\right),
$$

for any $X, Y \in \Gamma(T M), \xi \in \Gamma(\operatorname{RadTM})$ and $N \in \Gamma(\operatorname{ltr}(T M))$.

It is well known that for non degenerate submanifolds, the induced connection $\tilde{\nabla}$ is a metric connection. But this is not true incase of lightlike submanifolds(degenerate submanifolds). Indeed, considering $\overline{\widetilde{\nabla}}$ a metric connection, we have

$$
\left(\tilde{\nabla}_{X} g\right)(Y, Z)=\bar{g}\left(h^{l}(X, Y), Z\right)+\bar{g}\left(h^{l}(X, Z), Y\right),
$$

for any $X, Y, Z \in \Gamma(T M)$.

\subsection{Radical Transversal Lightlike Submanifold Of An Sasaki-Like Almost Contact Manifold With $B$-Metric}

In this section, we recall the definition of radical transversal lightlike submanifold of an Sasaki-like almost contact manifold with $B$-metric.

Definition 3.1.1 Let $\left(M, \tilde{g}, S(T M), S(T M)^{\perp}\right)$ be a lightlike submanifold of a Sasaki-like almost contact manifold with $B$-metric $(\bar{M}, \bar{\varphi}, \bar{g}, \overline{\widetilde{g}})$. Then $M$ is called radical transversal lightlike submanifold of $\bar{M}$ if

$$
\begin{gathered}
\bar{\varphi}(\operatorname{Rad} T M)=\operatorname{ltr}(T M), \\
\bar{\varphi}(S(T M))=S(T M) .
\end{gathered}
$$

Let $(M, \tilde{g})$ be a radical transversal lightlike submanifold of a Sasaki-like almost contact manifold with $B$-metric $(\bar{M}, \bar{\varphi}, \bar{g}, \overline{\widetilde{g}})$. Let $P$ and $S$ be the projection morphisms on radical distribution and screen distribution, respectively. Then for any $X \in \Gamma(T M)$, we have

$$
X=S X+P X
$$

where $S X \in \Gamma(S(T M))$ and $P X \in \Gamma(\operatorname{RadTM})$. Applying $\varphi$ to (3.1.3), we obtain

$$
\varphi X=T X+Q X
$$

where $T X$ and $Q X$ are the tangential and transversal components of $\varphi X$, respectively.

Similarly,

$$
\varphi U=t U+f U
$$

for any $U \in \Gamma(\operatorname{tr}(T M))$, where $t U$ and $f U$ denote the sections of $\Gamma(T M)$ and $\operatorname{tr}(T M)$ respectively.

Using the definition of Sasaki-like almost contact manifold with $B$-metric, the covariant derivatives of above tensor fields are defined as:

$$
\begin{gathered}
\left(\tilde{\nabla}_{X} T\right) Y=A_{Q Y} X+\varphi h^{l}(X, Y)-\tilde{g}(X, Y) \zeta-\eta(Y) X+2 \eta(X) \eta(Y) \zeta \\
h^{l}(X, T Y)+\tilde{\nabla}_{X}^{l} Q Y-Q \tilde{\nabla}_{X} Y=0 \\
h^{s}(X, T Y)+D^{s}(X, Q Y)-\varphi h^{s}(X, Y)=0 .
\end{gathered}
$$

In [11], Sahin investigated the integrability conditions for the screen distribution and radical distribution for a radical 
transversal lightlike submanifold of an indefinite Sasakian manifold. Here, we prove that the result also hold true for radical transversal lightlike submanifold of an indefinite Sasaki-like almost contact manifold with $B$-metric.

Lemma 3.1.2 Let $(M, \tilde{g})$ be a radical lightlike submanifold of a Sasaki-like almost contact manifold with $B$-metric $(\bar{M}, \bar{\varphi}, \eta, \zeta, \bar{g}, \overline{\widetilde{g}})$. Then we have

1. the screen distribution $S(T M)$ is integrable if and only if

$$
h^{l}(X, \varphi Y)=h^{l}(Y, \varphi X)
$$

for any $X, Y \in \Gamma(S(T M))$

2. the radical distribution $\operatorname{Rad}(T M)$ is integrable if and only if

$$
A_{Q X} Y=A_{Q Y} X
$$

for any $X, Y \in \Gamma(\operatorname{RadTM})$.

\subsection{Contact $C R$-submanifold Of An Sasaki-Like Almost Contact Manifold With $B$ - Metric}

Definition 3.2.1[13] A submanifold $(M, g)$ of a $(2 n+1)$-dimensional Sasaki-like almost contact manifold with $B$-metric $\bar{M}$ with structure tensors $(\varphi, \zeta, \eta)$ is called a contact $C R$-submanifold if there exists a differentiable distribution $D: x \rightarrow D_{x} \subseteq T_{x} M$ and the complementary orthogonal distribution $D^{\perp}: x \rightarrow D_{x}^{\perp} \subset T_{x} M$ on $M$ which satisfies the following conditions:

$\cdot \zeta \in D$,

- $\varphi D_{x} \subset T_{x} M$ for each $x \in M$,

- $\varphi D_{x}^{\perp} \subseteq T_{x} M^{\perp}$ for each point $x \in M$.

The complementary orthogonal distribution of $\varphi D^{\perp}$ in $T M^{\perp}$ is denoted by $\pi$. Then the tangent bundle $T \bar{M}$ of $\bar{M}$ has the following decomposition (for detail study on contact $C R$-submanifolds, see [12])

$$
T \bar{M}=T M \perp T M^{\perp}=T M \perp \bar{\varphi} D^{\perp} \perp \pi=D \perp D^{\perp} \perp \bar{\varphi} D^{\perp} \perp \pi .
$$

The usual definition of totally umbilical submanifolds does not work for submanifolds of indefinite Sasaki-like almost contact manifold with $B$-metric. Therefore, we need the following definition[13] :

Definition 3.2.2 Let $(M, g)$ be a contact $C R$-submanifold of a Sasaki-like almost contact manifold $(\bar{M}, \varphi, \zeta, \eta, \bar{g})$ with $B$-metric. Then $M$ is called a totally contact umbilical manifold if the second fundamental form of $M$ is of this form

$$
h(X, Y)=[g(X, Y)-\eta(X) \eta(Y)] \alpha+\eta(X) h(Y, \zeta)+\eta(Y) h(X, \zeta)
$$

for any vector fields $X, Y \in \Gamma(T M)$ and $\alpha \in \Gamma\left(T M^{\perp}\right)$. In particular, $\alpha=0$, implies that $(M, g)$ is a totally contact geodesic manifold. For contact $C R$-submanifold, we quote the following result[13]:

Lemma 3.2.3 Let $(M, g)$ be a totally contact umbilical $C R$-submanifold of a Sasaki-like almost contact manifold $(\bar{M}, \varphi, \zeta, \eta, \bar{g})$ with $B$-metric. Then $(M, g)$ is totally contact geodesic in $\bar{M}$ if $\operatorname{dim}\left(D^{\perp}\right)>2$.

\section{MAIN RESULTS}

The above definition also hold true for a lightlike submanifold $(M, \tilde{g})$. A lightlike submanifold of an indefinite Sasaki-like almost contact manifold with $B$-metric is said to be totally umbilical if, for any $X, Y \in(\mathrm{M})$, 


$$
\begin{aligned}
& h^{l}(X, Y)=[\tilde{g}(X, Y)-\eta(X) \eta(Y)] \alpha_{l}+\eta(X) h^{l}(Y, \zeta)+\eta(Y) h^{l}(X, \zeta) \\
& h^{s}(X, Y)=[\tilde{g}(X, Y)-\eta(X) \eta(Y)] \alpha_{s}+\eta(X) h^{s}(Y, \zeta)+\eta(Y) h^{s}(X, \zeta)
\end{aligned}
$$

where $\alpha_{s} \in S(T M)^{\perp}$ and $\alpha_{l} \in \operatorname{ltr}(T M)$.

Theorem 4.1 Let $(M, \tilde{g})$ be a totally contact umbilical radical transversal lightlike submanifold and $(M, g)$ be a totally contact umbilical $C R$-submanifold of a Sasaki-like almost contact manifold with $B$-metric $(\bar{M}, \bar{\varphi}, \eta, \zeta, \bar{g}, \overline{\widetilde{g}})$. Then the holomorphic distribution $D$ of totally contact umbilical $C R$-submanifold $(M, g)$ is integrable if and only if the screen distribution $S(T M)$ of radical transversal lightlike submanifold $(M, \tilde{g})$ is integrable.

Proof. Let $X, Y \in \Gamma(D)$ and $N \in \Gamma\left(\varphi(D)^{\perp}\right)$ then using (2.1.2), (2.1.3) and (2.2.4) , we have

$$
\begin{aligned}
& \bar{g}([X, Y], \varphi N)=\overline{\widetilde{g}}([X, Y], N) \\
& =\overline{\widetilde{g}}\left(h^{l}(X, Y)-h^{l}(Y, X), N\right)
\end{aligned}
$$

where $X, Y \in \Gamma(S(T M))$ and $N \in \Gamma(\operatorname{ltr}(T M))$. Further, using the definition of totally contact umbilical radical transversal lightlike submanifold, we have

$$
\bar{g}([X, Y], \varphi N)=(-\tilde{g}(X, Y)+\tilde{g}(Y, X)) \tilde{g}\left(\alpha_{l}, N\right)=0
$$

and hence the above result follows.

Theorem 4.2 Let $(M, \tilde{g})$ be a totally contact umbilical radical transversal lightlike submanifold and $(M, g)$ be a totally contact umbilical $C R$-submanifold of a Sasaki-like almost contact manifold with $B$-metric $(\bar{M}, \bar{\varphi}, \eta, \zeta, \bar{g}, \overline{\tilde{g}})$. Then, $\alpha_{s}=0$.

Proof. For any $X \in \Gamma(S(T M)-\zeta)$ and using (2.1.11) and (3.1.6), we have

$$
\begin{aligned}
& \tilde{g}(X, X) \zeta=-\left(\nabla_{X} \varphi X+h^{l}(X, \varphi X)+h^{s}(X, \varphi X)\right) \\
& +\left(T \nabla_{X} X+Q \nabla_{X} X+\varphi h^{l}(X, X)+\varphi h^{s}(X, X)\right) .
\end{aligned}
$$

Comparing the screen transversal parts of above equation, we get

$$
h^{s}(X, \varphi X)=\varphi h^{s}(X, X)
$$

further using the definition of totally contact umbilical radical transversal lightlike submanifold, we have

$$
\tilde{g}(X, X) \tilde{g}\left(\alpha_{s}, \varphi W\right)=0 .
$$

then the non degeneracy of $S(T M)$ and $S\left(T M^{\perp}\right)$ implies that $\alpha_{s}=0$.

Theorem 4.3 Let $(M, \tilde{g})$ be a totally contact umbilical radical transversal lightlike submanifold and $(M, g)$ be a totally contact umbilical $C R$-submanifold of a Sasaki-like almost contact manifold with $B$-metric $(\bar{M}, \bar{\varphi}, \eta, \zeta, \bar{g}, \overline{\widetilde{g}})$. Then $\alpha_{l}=0$.

Proof. Let $(M, g)$ be a totally contact umbilical $C R$-submanifold and let $X \in \varphi(\pi)$ then using the definition of Sasaki-like almost contact manifold with $B$-metric, we have

$$
\bar{\nabla}_{X} \varphi X=\varphi \bar{\nabla}_{X} X+\left(\bar{\nabla}_{X} \varphi\right) X
$$

Taking the tangent components of above equation, we obtain

$$
g(X, X) \zeta=A_{\varphi X} X+\varphi \nabla_{X} X+\varphi h^{1}(X, X)+\varphi h^{2}(X, X)
$$


for any $Y \in \Gamma(D)$

$$
\left(A_{\varphi X} X+\varphi h^{1}(X, X), \varphi Y\right)=0
$$

using (2.1.3), we have

$$
\overline{\widetilde{g}}\left(A_{\varphi X} X+\varphi h^{l}(X, X), Y\right)=0 .
$$

from (2.2.7), we get

$$
\left.\overline{\widetilde{g}}\left(h^{s}(X, Y), \varphi X\right)+h^{l}(X, X), \varphi Y\right)=0 .
$$

using (4.1)

$$
\overline{\widetilde{g}}(X, X) \overline{\widetilde{g}}\left(\alpha_{l}, \varphi Y\right)=0
$$

the above equation holds true if and only if $\alpha_{l}=0$.

Theorem 4.4 Let $(M, \tilde{g})$ be a totally contact umbilical radical transversal lightlike submanifold and $(M, g)$ be a totally contact umbilical $C R$-submanifold of a Sasaki-like almost contact manifold with $B$-metric $(\bar{M}, \bar{\varphi}, \eta, \zeta, \bar{g}, \overline{\widetilde{g}})$. then $(M, \tilde{g})$ is a totally contact geodesic radical transversal lightlike submanifold.

Proof. Using Theorem 4.2 and Theorem 4.3, the result holds true.

Theorem 4.5 Let $(M, \tilde{g})$ be a totally contact umbilical radical transversal lightlike submanifold and $(M, g)$ be a totally contact umbilical $C R$-submanifold of a Sasaki-like almost contact manifold with $B$-metric $(\bar{M}, \bar{\varphi}, \eta, \zeta, \bar{g}, \overline{\widetilde{g}})$ such that $\overline{\widetilde{\nabla}}_{X} \zeta \in \Gamma(T M)$ then the induced connection $\tilde{\nabla}$ becomes a metric connection on $(M, \tilde{g})$.

Proof. Using Theorem 4.3, we have $\alpha_{l}=0$. Since $\bar{\nabla}_{X} \zeta \in \Gamma(T M)$ implies $h^{l}(X, \zeta)=0$ therefore using definition of totally contact umbilical submanifold, we obtain

$$
h^{l}=0
$$

then using Theorem 2.2 in [[4], page159], the induced connection $\tilde{\nabla}$ becomes a metric connection on $(M, \tilde{g})$.

Theorem 4.6 Let $(M, \tilde{g})$ be a totally contact umbilical radical transversal lightlike submanifold and $(M, g)$ be a totally contact umbilical $C R$-submanifold of a Sasaki-like almost contact manifold with $B$-metric $(\bar{M}, \bar{\varphi}, \eta, \zeta, \bar{g}, \overline{\widetilde{g}})$ such that $\overline{\widetilde{\nabla}}_{X} \zeta \in \Gamma(T M)$ then $(M, \tilde{g})$ becomes a totally geodesic radical transversal lightlike submanifold.

Proof. Using Theorem 4.2, we have $\alpha_{s}=0$. Since $\bar{\nabla}_{X} \zeta \in \Gamma(T M)$ implies $h^{s}(X, \zeta)=0$ therefore using definition of totally contact umbilical submanifold, we obtain

$$
h^{s}=0 \text {, }
$$

Then using (4.6) and (4.7), the result follows.

\section{ACKNOWLEDGMENTS}

Our thanks to the anonymous referee for his/her comments who have helped us to improve this paper.

\section{References}

[1] Bejancu A., $C R$-submanifolds of a Kaehler manifold-I, Proc. Amer. Math. Soc., 69 (1978), 135-142.

[2] Bejancu A., CR -submanifolds of a Kaehler manifold-II, Trans. Amer. Math. Soc., 250 (1979), 333-345.

[3] Blair D. E., Riemannian Geometry of Contact and Symplectic Manifolds. Progress in Mathematics 203, Birkhauser, 
Boston (2002).

[4] Duggal K. L., Bejancu A. , Lightlike Submanifolds of Semi-Riemannian Manifolds and Applications, 364 of Mathematics and its Applications, Kluwer Academic Publishers, Dordrecht, The Netherlands, (1996).

[5] Duggal K. L. and Jin D. H., Totally umbilical lightlike submanifolds, Kodai Math. J., 26 (2003), 49-68.

[6] Duggal K. L. and Sahin B., Lightlike submanifolds of indefinite Sasakian manifolds, Int. Jour. Math and Math. Sci., 26 (2007), 49-68.

[7] Ganchev, G., Mihova,V., Gribachev, K., Almost contact manifolds with B-metric, Math. Balkanica (N.S.), 7(3-4) (1993), 261-276. manifolds with B-metric, Publ Inst. Math.(N.S), 42 (1987), 107-121.

[8] Matsumoto K., On contact CR-submanifolds of Sasakian manifolds, International J. Math. Math. Sci., 6(2)(1983), 313-326.

[9] Ivanov S., Manev H. and Manev M., Sasaki-like Almost contact complex Riemannian manifolds, J. Geom and Physics, 107 (2016), 136-148.

[10] Nakova G., Some lightlike submanifolds of almost complex manifolds with Norden metric, J. Geometry, 103 (2012), 293-312.

[11] Yildirim C. and Sahin B., Transversal lightlike submanifolds of indefinite Sasakian manifolds, Turk J Math., 34 (2010), 561-583.

[12] Yano K. and Kon M., Differential geometry of CR submanifolds, Geometria Dedicata, 10(1981), 369-391.

[13] Yano K. and Kon M., CR submanifolds of Kaehlerian and Sasakian manifolds, World Scientific, (1984).

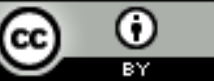

This work is licensed under a Creative Commons Attribution 4.0 International License. 